Mathematical Research Letters 1, 559-563 (1994)

\title{
GLOBAL ANALYTIC HYPOELLIPTICITY IN THE PRESENCE OF SYMMETRY
}

\author{
Michael Christ
}

A linear partial differential operator, $L$, is said to be globally analytic hypoelliptic on some real analytic manifold $M$ without boundary if, for any $u \in \mathcal{D}^{\prime}(M)$ such that $L u \in C^{\omega}(M)$, one has $u \in C^{\omega}(M)$. It is of some interest to determine under what circumstances this property holds, especially for sums of squares of vector fields satisfying the bracket hypothesis of Hörmander, and for related operators such as $\square_{b}$ arising in complex analysis in several variables. Examples are known (and discussed at the end of this note) in which global analytic hypoellipticity holds [C1], [C2], [CoH], [DT1], [DT2], yet analytic hypoellipticity in the more usually studied local sense fails [Ch1], [Ch2], [HH]. Those examples all possess certain global symmetries. We have recently shown [Ch3] that global analytic hypoellipticity does not always hold for certain of these operators, so that symmetry hypotheses are no mere crutch eventually to be discarded, as might have been hoped. Our aim here is to show how global analytic hypoellipticity follows quite directly from the presence of certain symmetry, emphasizing the general nature of the result and structure of the argument.

Throughout this note, "analytic" means "real analytic", and is denoted by $C^{\omega}$. The symbols $L^{2}, C^{\infty}, C^{\omega}$ refer to sections of whichever vector bundle is relevant. Our results are formulated for sections of vector bundles, rather than for functions, because that situation arises for $\square_{b}$, but the reader will lose no ideas by assuming all functions to be scalar-valued.

Let there be given an analytic manifold $\Omega$ without boundary, not necessarily compact, a compact subset $K \subset \Omega$, and a linear partial differential operator $L$ mapping sections of one analytic vector bundle over $\Omega$ to sections of a second such bundle. We consider the following notion of regularity.

Received May 24, 1994.

Research supported by the National Science Foundation. 
Definition. $L$ is globally analytic hypoelliptic relative to the pair $(\Omega, K)$ if, whenever $u \in \mathcal{D}^{\prime}(\Omega), L u \in C^{\omega}(\Omega)$ and $u \in C^{\omega}(\Omega \backslash K)$, then necessarily $u \in C^{\omega}(\Omega)$.

When $\Omega$ is a compact manifold and $K=\Omega$, this reduces to the usual notion of global regularity. An interesting example in which this type of regularity fails, for $K$ a single point, is in [M1], while other examples are (implicit) in [Ch3].

Let $G$ be a compact, connected Lie group. Let $M$ be an analytic manifold, without boundary, equipped with the structure of an analytic principal fiber bundle with structure group $G$. Thus $G$ acts on $M$, the quotient is an analytic manifold, and the fibers of the quotient map may be identified with $G$. Let $E_{1}, E_{2}$ be analytic vector bundles over $M$, to which the $G$ action is assumed to lift as invertible (analytic) endomorphisms. Let $L$ be a linear partial differential operator on $M$, with $C^{\omega}$ coefficients, mapping sections of $E_{1}$ to sections of $E_{2}$.

Let $T G \subset T M$ be the subbundle tangent to the $G$-orbits. Denote by $\Sigma \subset T^{*} M \otimes E_{1}$ the characteristic variety on which the principal symbol of $L$ fails to be invertible, and by $G^{\perp} \subset T^{*} M \otimes E_{1}$ the annihilator of the subbundle $T G \otimes E_{1}^{*}$. Given any compact subset of $M$, we write $\|f\|_{H^{t}}$ for some fixed Sobolev-type norm of order $t \geq 0$ on all $C^{\infty}$ functions supported in that subset.

Our hypotheses are:

(1) $L$ commutes with the action of $G$.

(2) $L$ is $C^{\infty}$ hypoelliptic.

(3) $L$ is transversally elliptic, that is, $\Sigma \cap G^{\perp}=M \times\{0\}$.

(4) There exist $\varepsilon, s \in \mathbb{R}^{+}$such that for any compactly supported functions $\eta, \tilde{\eta} \in C^{\infty}$ such that $\tilde{\eta} \cdot \eta \equiv \eta$, there exists $C<\infty$ such that for any smooth function $u$,

$$
\|\eta u\|_{H^{\varepsilon}} \leq C\|\tilde{\eta} L u\|_{H^{s}}+C\|\tilde{\eta} u\|_{L^{2}}
$$

Theorem. Under the above hypotheses, $L$ is globally analytic hypoelliptic relative to $(M, K)$, for any $G$-invariant compact subset $K$ of $M$.

Variations on this set of hypotheses are possible, as will be apparent from the proof. For instance, (3) may be replaced by the weaker assumption that $L$ is microlocally analytic hypoelliptic in some conic neighborhood of $G^{\perp}$. When $K=M$, (4) may be replaced by the assumption that there exists $s \in \mathbb{R}^{+}$such that, for all smooth $u,\|u\|_{L^{2}} \leq C\|L u\|_{H^{s}}$.

A device useful in the proof will be a bi-invariant orthogonal projection $P: L^{2}(G) \mapsto L^{2}(G)$, such that for any function $f$ belonging to the range 
of $P,\|f\|_{L^{2}(G)} \leq \delta\|f\|_{H^{\varepsilon}(G)}$. For any $\varepsilon, \delta>0$, there exists such a projection. Indeed, fixing a large constant $\lambda$ and a bi-invariant Laplace-Beltrami operator $A$ on $G$, define $I-P$ to be the orthogonal projection of $L^{2}(G)$ onto the direct sum of all eigenspaces associated to eigenvalues of $A$ not exceeding $\lambda$. Then for any function, $f=(-A+I)^{-\varepsilon / 2} \circ(-A+I)^{\varepsilon / 2} f$, so that $\|f\|_{H^{\varepsilon}} \sim\left\|(-A+I)^{\varepsilon / 2} f\right\|_{L^{2}}$; if $f$ belongs to the range of $P$, then this last quantity is $\geq \lambda^{\varepsilon / 2}\|f\|_{L^{2}}$.

Since $A$ is elliptic, its eigenfunctions are all real analytic, and hence $I-P$ maps $L^{2}(G)$ continuously to $C^{\omega}(G) . P$ is bi-invariant since $A$ is, and hence (or because $I-P$ is $C^{\omega}$-regularizing) preserves $C^{\omega}(G)$. The $G$-action transfers $P$ to an operator $Q$ acting on sections of any $G$-invariant vector bundle over $M$, which preserves $C^{\infty}$ and $C^{\omega}$. Since $I-P$ may be realized as convolution, on either the left or the right, with a function on $G$, and since $L$ commutes with the action of $G, Q$ commutes with $L$.

Fix a basis $\left\{X_{j}\right\}$ for the Lie algebra of left-invariant vector fields on $G$; these will be regarded also as operators acting on functions defined on $M$, via the action of $G$. Denote by $X^{\alpha}$ any finite composition, with $|\alpha|$ factors, of any of the $X_{j}$.

For any $t>0$, denote by $\tilde{H}^{t}$ the Sobolev space of functions in $L^{2}(M)$ which possess $t$ derivatives along the fibers.

Proof of Theorem. Suppose that $L u \in C^{\omega}(M)$. $u$ is $C^{\infty}$ since $L$ is assumed to be $C^{\infty}$ hypoelliptic, so the restriction of $(I-Q) u$ to each fiber is $C^{\omega}$, uniformly in the fiber, and consequently the analytic wave front set of $(I-Q) u$ is contained in $G^{\perp}$. On the other hand, since $L((I-Q) u)=$ $(I-Q) L u \in C^{\omega}$, the analytic wave front set must be contained in the characteristic variety $\Sigma$ of $L$. We have assumed $G^{\perp} \cap \Sigma$ to be empty, so $(I-Q) u$ is analytic.

Set $g=Q u$. Then $L g=Q L u \in C^{\omega}(M)$, and moreover, $g \in C^{\omega}(M \backslash K)$ since $u$ is $C^{\omega}$ there, and since $Q$ preserves $C^{\omega}$ in any open $G$-invariant set. Consider any $\alpha$. Since $L$ commutes with the action of $G$, it commutes with $X^{\alpha}$. Then for any fixed $N$, and any relatively compact $U \subset M$, $\left\|L\left(X^{\alpha} g\right)\right\|_{C^{N}(U)}=\left\|X^{\alpha} L g\right\|_{C^{N}(U)} \leq C^{1+|\alpha|}|\alpha|^{|\alpha|}$, uniformly in $\alpha$.

Fix compactly supported, $G$-invariant $C^{\infty}$ functions $\eta, \tilde{\eta}$, such that $\tilde{\eta} \cdot \eta \equiv$ $\eta$ and $\eta \equiv 1$ in a neighborhood of $K$. By (4), then,

$$
\left\|\eta X^{\alpha} g\right\|_{H^{\varepsilon}} \leq C^{1+|\alpha|}|\alpha|^{|\alpha|}+C^{\prime}\left\|\tilde{\eta} X^{\alpha} g\right\|_{L^{2}},
$$

where $C^{\prime}$ is independent of the choices made in the construction of $Q$. Now

$$
\left\|\tilde{\eta} X^{\alpha} g\right\|_{L^{2}} \leq C^{1+|\alpha|}|\alpha|^{|\alpha|}+\left\|\eta X^{\alpha} g\right\|_{L^{2}}
$$


since $g \in C^{\omega}(M \backslash K)$. Since $P$ is bi-invariant and $g$ is in the range of $Q$, $X^{\alpha} g$ is also in the range of $Q$, and consequently $\left\|\eta X^{\alpha} g\right\|_{L^{2}} \leq \delta\left\|\eta X^{\alpha} g\right\|_{\tilde{H}^{\varepsilon}}$, where $Q$ may be constructed so that $\delta$ is as small as we please. Putting this all together,

$$
\left\|\eta X^{\alpha} g\right\|_{H^{\epsilon}} \leq C^{1+|\alpha|}|\alpha|^{|\alpha|}+\delta C^{\prime}\left\|\eta X^{\alpha} g\right\|_{\tilde{H}^{\epsilon}} .
$$

Choosing $\delta$ to be sufficiently small and then absorbing the second term from the right-hand side into the left, we conclude that $X^{\alpha} g=O\left(C^{|\alpha|}|\alpha|^{|\alpha|}\right)$, in the $\tilde{H}^{\varepsilon}$ norm and hence in the $L^{2}$ norm, in a neighborhood of $K$.

The analytic wave front set of $g$ is therefore contained in $G^{\perp}$. As in the discussion of $(I-Q) u$, this implies that $g$, hence $u$, is analytic.

Our results are by no means definitive; there should for instance be generalizations to boundary value problems, and generalizations which are simultaneously microlocal and global in character.

Here are some examples satisfying our hypotheses. In all of these, $M$ is a compact manifold without boundary, and $K=\emptyset$. In all except example (4), $L$ maps scalar-valued functions to scalar-valued functions.

(1) Let $M$ be the torus $\mathbb{T}^{3}$ with coordinates $(x, y, t)$, and take $L=$ $\partial_{x}^{2}+\left(\partial_{y}-\theta(x) \partial_{t}\right)^{2}$, where $\theta$ is periodic and real-valued, and has the property that for each $x$ there exists $k>0$ such that $d^{k} \theta(x) / d x^{k} \neq$ 0 . Regarding $\mathbb{T}^{3}$ as a principal fiber bundle with structure group $\mathbb{T}$, acting in the $t$ variable, all hypotheses of the Theorem are satisfied. However, if there exists $x_{0}$ such that $d \theta\left(x_{0}\right) / d x=0$, then $L$ fails to be analytic hypoelliptic in the local sense [Ch2], [Ch4].

(2) Let $G$ be any compact Lie group and $\left\{X_{j}\right\}$ a collection of leftinvariant vector fields on $G$ which generate its Lie algebra. Take $M=G$, and $L=\sum_{j} X_{j}^{2}$. Again, all hypotheses of the Theorem are fulfilled. But if the $X_{j}$ are independent, if the number of $X_{j}$ is one less than the dimension of $G$, and if that dimension is even, then $L$ fails to be analytic hypoelliptic in the local sense [M2].

(3) Fix a compact analytic manifold $M^{\prime}$ without boundary and a compact Lie group $G$, and set $M=M^{\prime} \times G$. Fix left-invariant vector fields $\left\{\tilde{Y}_{j}\right\}$ on $G$ which generate its Lie algebra. Letting $G$ act on $M$ by left multiplication in the second factor of the product, these induce corresponding vector fields $Y_{j}$ on $M$. Fix analytic vector fields $\tilde{Z}_{j}$ on $M^{\prime}$ which span its tangent space at every point, and denote by $Z_{j}$ the corresponding vector fields on $M$. Fix analytic, real-valued functions $\theta_{j}$ on $M^{\prime}$, regarding these also as functions defined on $M$ and independent of the second factor, and finally set $X_{j}=Z_{j}+\varepsilon \theta_{j} Y_{j}$ and $L_{\varepsilon}=\sum_{j} X_{j}^{2}$, where $\varepsilon \in \mathbb{R}$ is a parameter. 
All hypotheses of our Theorem are satisfied, provided that the Lie algebra generated by the $X_{j}$ spans the tangent space to $M$ at every point. Many examples can be constructed in which this holds for generic $\varepsilon \in \mathbb{R}$.

(4) Let $\Omega \subset \mathbb{C}^{n}$ be a bounded, real analytic, circular domain (that is, if $z \in \Omega$ and $\theta \in \mathbb{R}$ then $\left.\left(e^{i \theta} z_{1}, \ldots e^{i \theta} z_{n}\right) \in \Omega\right)$. Let $M=\partial \Omega$ and take $G$ to be the circle group $\mathbb{T}=\left\{e^{i \theta}\right\}$. Assume that the orbits of $G$ are transverse to the complex tangent space of $M$. Let $L=\square_{b}$ be the Kohn Laplacian, mapping smooth $(p, q)$ forms on $M$ to $(p, q)$ forms on $M$. Then the hypotheses of our Theorem are satisfied, provided that $L$ is subelliptic, which holds under a standard hypothesis of finite type.

\section{References}

[C1] S. C. Chen, Global analytic hypoellipticity of the $\bar{\partial}-$ Neumann problem on circular domains, Invent. Math. 92 (1988), 173-185.

[C2] , Real analytic regularity of the Szegö projection on circular domains, Pacific J. Math. 148 (1991), 225-235.

[Ch1] M. Christ, Analytic hypoellipticity breaks down for weakly pseudoconvex Reinhardt domains, International Math. Research Notices 1 (1991), 31-40.

[Ch2] _ A necessary condition for analytic hypoellipticity, Math. Research Letters 1 (1994), 241-248.

[Ch3] - The Szegö projection need not preserve global analyticity, preprint.

[Ch4] , A class of hypoelliptic PDE admitting non-analytic solutions, Contemporary Mathematics 137 (1992), 155-168.

$[\mathrm{CoH}]$ P. D. Cordaro and A. A. Himonas, Global analytic hypoellipticity of a class of degenerate elliptic operators on the torus, Math. Research Letters 1 (1994), 501510.

[DT1] M. Derridj and D. S. Tartakoff, Global analyticity for $\square_{b}$ on three dimensional pseudoconvex CR manifolds, preprint.

[DT2] Microlocal analyticity for the canonical solution to $\bar{\partial}_{b}$ on some rigid weakly pseudoconvex hypersurfaces in $\mathbb{C}^{2}$, preprint.

[HH] N. Hanges and A. A. Himonas, Singular solutions for sums of squares of vector fields, Comm. Partial Differential Equations 16 (1991), 1503-1511.

[M1] G. Métivier, Non-hypoellipticité analytique pour $D_{x}^{2}+\left(x^{2}+y^{2}\right) D_{y}^{2}$, Comptes Rendus Acad. Sci. Paris 292 (1981), 401-404.

[M2] Une classe d'opérateurs non hypoélliptiques analytiques, Indiana Math. J. 29 (1980), 823-860.

Department of Mathematics, University of California, Los Angeles, Los Angeles, CA $90024-1555$.

E-mail address: christ@math.ucla.edu 